\title{
Treewidth of the Line Graph of a Complete Graph
}

\author{
Daniel J. Harvey ${ }^{1}$ and David R. Wood ${ }^{2}$ \\ ${ }^{1}$ DEPARTMENT OF MATHEMATICS AND STATISTICS \\ THE UNIVERSITY OF MELBOURNE \\ PARKVILLE, VICTORIA, 3010, AUSTRALIA \\ E-mail: d.harvey@pgrad.unimelb.edu.au \\ ${ }^{2}$ SCHOOL OF MATHEMATICAL SCIENCES \\ MONASH UNIVERSITY \\ CLAYTON, VICTORIA, 3800, AUSTRALIA \\ E-mail: david.wood@monash.edu
}

Received October 28, 2012; Revised April 22, 2014

Published online in Wiley Online Library (wileyonlinelibrary.com).

DOI 10.1002/jgt.21813

\begin{abstract}
In recent articles by Grohe and Marx, the treewidth of the line graph of a complete graph is a critical example-in a certain sense, every graph with large treewidth "contains" $L\left(K_{n}\right)$. However, the treewidth of $L\left(K_{n}\right)$ was not determined exactly. We determine the exact treewidth of the line graph of a complete graph. (c) 2014 Wiley Periodicals, Inc. J. Graph Theory 00: 1-7, 2014
\end{abstract}

\section{INTRODUCTION}

The treewidth $\operatorname{tw}(G)$ of a graph $G$ is a graph invariant used to measure how "tree-like" $G$ is. It is of particular importance in structural and algorithmic graph theory; see the surveys $[1,5]$. The treewidth $\operatorname{tw}(G)$ is the minimum width of a tree decomposition of $G$, which is defined as follows:

Contract grant sponsor: Australian Postgraduate Award (to D.J.H.); Contract grant sponsor: Australian Research Council (to D.W.).

Journal of Graph Theory

(C) 2014 Wiley Periodicals, Inc. 
Definition. A tree decomposition of a graph $G$ is a pair $\left(T,\left\{A_{x} \subseteq V(G): x \in V(T)\right\}\right)$ such that:

- $T$ is a tree.

- $\left\{A_{x} \subseteq V(G): x \in V(T)\right\}$ is a collection of sets of vertices of $G$, each called a bag, indexed by the nodes of $T$.

- For all $v \in V(G)$, the nodes of $T$ indexing the bags containing $v$ induce a nonempty (connected) subtree of $T$.

- For all $v w \in E(G)$, there exists a bag of $T$ containing both $v$ and $w$.

The width of a tree decomposition is the maximum size of a bag of $T$, minus 1 . This minus 1 is added to ensure that every tree has treewidth 1 . Similarly, define the pathwidth of a graph $G$, denoted $\operatorname{pw}(G)$, to be the minimum width of a tree decomposition where the underlying tree is a path. (We call such a tree decomposition a path decomposition.) It follows from the definition that $\operatorname{pw}(G) \geqslant \operatorname{tw}(G)$ for all graphs $G$.

The line-graph $L(G)$ of a graph $G$ is the graph with $V(L(G))=E(G)$, such that two vertices of $L(G)$ are adjacent when the corresponding edges of $G$ are incident at a vertex.

In recent articles by Marx [4] and Grohe and Marx [3], the treewidth of the line graph of a complete graph is a critical example. For a graph $G$, let $G^{(q)}$ denote the graph created by replacing each vertex of $G$ with a clique of size $q$ and replacing each edge between two vertices with all of the edges between the two new cliques. Marx [4] shows that if $\operatorname{tw}(G) \geqslant k$, then $G^{(p)}$ contains $L\left(K_{k}\right)^{(q)}$ as a minor (for appropriate choices of $p$ and $q$, depending on $k$ and $|V(G)|)$. Then Grohe and Marx [3] show that $\operatorname{tw}\left(L\left(K_{n}\right)\right) \geqslant \frac{\sqrt{2}-1}{4} n^{2}+O(n)$. In this article, we determine $\operatorname{tw}\left(L\left(K_{n}\right)\right)$ exactly. As it turns out, the minimum width tree decomposition that we construct is also a path decomposition. Hence, we prove the following result.

\section{Theorem 1.}

$$
\operatorname{tw}\left(L\left(K_{n}\right)\right)=\operatorname{pw}\left(L\left(K_{n}\right)\right)= \begin{cases}\left(\frac{n-1}{2}\right)\left(\frac{n-1}{2}\right)+n-2, & \text { if } n \text { is odd } \\ \left(\frac{n-2}{2}\right)\left(\frac{n}{2}\right)+n-2, & \text { if } n \text { is even }\end{cases}
$$

Note the following conventions: if $S$ is a subgraph of a graph $G$ and $x \in V(G)-V(S)$, then let $S \cup\{x\}$ denote the subgraph of $G$ with vertex set $V(S) \cup\{x\}$ and edge set $E(S) \cup$ $\{x y: y \in V(S), x y \in E(G)\}$. Similarly, if $u \in V(S)$, let $S-\{u\}$ denote the subgraph with vertex set $V(S)-\{u\}$ and edge set $E(S)-\{u w: w \in V(S)-\{u\}\}$.

\section{LINE-BRAMBLES AND THE TREEWIDTH DUALITY THEOREM}

A bramble of a graph $G$ is a collection $\mathcal{B}$ of connected subgraphs of $G$ such that each pair of subgraphs $X, Y \in \mathcal{B}$ touch. Subgraphs $X$ and $Y$ touch when they either have at least one vertex in common, or there exists an edge in $G$ with one end in $V(X)$ and the other in $V(Y)$. The order of a bramble is the size of the smallest hitting set $H$, where a hitting set of a bramble $\mathcal{B}$ is a set of vertices $H$ such that $H \cap V(X) \neq \emptyset$ for all $X \in \mathcal{B}$. For a given graph $G$, the bramble number bn $(G)$ is the maximum order of a bramble of $G$. Brambles are important due to the following theorem of Seymour and Thomas [6]:

Theorem 2. (Treewidth Duality Theorem) For every graph $G, \operatorname{bn}(G)=\operatorname{tw}(G)+1$. 
In this article we employ the following standard approach for determining the treewidth and pathwidth of a particular graph $G$. First construct a bramble of large order, thus proving a lower bound on $\operatorname{tw}(G)$. Then to prove an upper bound, construct a path decomposition of small width. Given that $\operatorname{tw}(G) \leqslant \operatorname{pw}(G)$, this is sufficient to prove Theorem 1.

In order to construct a bramble of the line graph $L(G)$, define the following:

Definition. A line-bramble $\mathcal{B}$ of $G$ is a collection of connected subgraphs of $G$ satisfying the following properties:

- For all $X \in \mathcal{B},|V(X)| \geqslant 2$.

- For all $X, Y \in \mathcal{B}, V(X) \cap V(Y) \neq \emptyset$.

Define a hitting set for a line-bramble $\mathcal{B}$ to be a set of edges $H \subseteq E(G)$ that intersects each $X \in \mathcal{B}$. Then define the order of $\mathcal{B}$ to be the size of the minimum hitting set $H$ of $\mathcal{B}$.

Lemma 3. Given a line-bramble $\mathcal{B}$ of $G$, there is a bramble $\mathcal{B}^{\prime}$ of $L(G)$ of the same order.

Proof. Given a line-bramble $\mathcal{B}$, define $\mathcal{B}^{\prime}:=\{L(G)[E(X)]: X \in \mathcal{B}\}$. Let $X \in \mathcal{B}$. Since $X$ is connected and $|V(X)| \geqslant 2$, the subgraph $X$ contains an edge. So $E(X)$ induces a nonempty connected subgraph of $L(G)$. Consider $E(X)$ and $E(Y)$ in $\mathcal{B}^{\prime}$. Thus $V(X) \cap$ $V(Y) \neq \emptyset$. Let $v$ be a vertex in $V(X) \cap V(Y)$. Then there exists some $x v \in E(X)$ and $v y \in E(Y)$, and thus in $L(G)$ there is an edge between the vertex $x v$ and the vertex $v y$. Hence $E(X)$ and $E(Y)$ touch, and so $\mathcal{B}^{\prime}$ is a bramble of $L(G)$. All that remains is to ensure $\mathcal{B}$ and $\mathcal{B}^{\prime}$ have the same order. If $H$ is a minimum hitting set for $\mathcal{B}$, then $H$ is also a set of vertices in $L(G)$ that intersects a vertex in each $E(X) \in \mathcal{B}^{\prime}$. So $H$ is a hitting set for $\mathcal{B}^{\prime}$ of the same size. Conversely, if $H^{\prime}$ is a minimum hitting set of $\mathcal{B}^{\prime}$, then $H^{\prime}$ is a set of edges in $G$ that contains an edge in each $X \in \mathcal{B}$. So $H^{\prime}$ is a hitting set for $\mathcal{B}$. Thus, the orders of $\mathcal{B}$ and $\mathcal{B}^{\prime}$ are equal.

Hence, in order to determine a lower bound on the bramble number bn $(L(G))$, it is sufficient to construct a line-bramble of $G$ of large order. We will now define a particular line-bramble for any graph $G$ with $|V(G)| \geqslant 3$.

Definition. Given a graph $G$ and a vertex $v \in V(G)$, the canonical line-bramble for $v$ of $G$ is the set of connected subgraphs $X$ of $G$ such that either $|V(X)|>\frac{|V(G)|}{2}$, or $|V(X)|=\frac{|V(G)|}{2}$ and $X$ contains $v$. Note that if $|V(G)|$ is odd, then no elements of the second type occur.

Lemma 4. For every graph $G$ with $|V(G)| \geqslant 3$ and for all $v \in V(G)$, the canonical line-bramble for $v$, denoted by $\mathcal{B}$, is a line-bramble of $G$.

Proof. By definition, each element of $\mathcal{B}$ is a connected subgraph. Since $|V(G)| \geqslant 3$, each element of $\mathcal{B}$ contains at least two vertices. All that remains to show is that each pair of subgraphs $X, Y$ in $\mathcal{B}$ intersect in at least one vertex. If $|V(X)|=|V(Y)|=\frac{|V(G)|}{2}$, then $X$ and $Y$ intersect at $v$. Otherwise, without loss of generality, $|V(X)|>\frac{|V(G)|}{2}$ and $|V(Y)| \geqslant$ $\frac{|V(G)|}{2}$. If $V(X) \cap V(Y)=\emptyset$, then $|V(X) \cup V(Y)|=|V(X)|+|V(Y)|>|V(G)|$, which is a contradiction.

Let $v \in V(G)$ be an arbitrary vertex and let $H$ be a minimum hitting set of $\mathcal{B}$, the canonical line-bramble for $v$. Consider the graph $G-H$. Since $H$ is a set of edges, 
$V(G-H)=V(G)$. Then each component of $G-H$ contains at most $\frac{|V(G)|}{2}$ vertices, otherwise some component of $G-H$ contains an element of $\mathcal{B}$ that does not contain an edge of $H$. Similarly, if a component contains $\frac{|V(G)|}{2}$ vertices, it cannot contain the vertex $v$. Thus, our hitting set $H$ must be large enough to separate $G$ into such components. The next lemma follows directly:

Lemma 5. Let $G$ be a graph with $|V(G)| \geqslant 3$, let $v$ be a vertex of $G$, and let $\mathcal{B}$ be the canonical line-bramble for $v$. Then $H \subseteq E(G)$ is a hitting set of $\mathcal{B}$ if and only if every component of $G-H$ has at most $\frac{|V(G)|}{2}$ vertices, and $v$ is not in a component of $G-H$ that contains exactly $\frac{|V(G)|}{2}$ vertices.

Note the similarity between this characterization and the bisection width of a graph (see [2], for example), which is the minimum number of edges between any $A, B \subset V(G)$ where $A \cap B=\emptyset$ and $|A|=\left\lfloor\frac{|V(G)|}{2}\right\rfloor$ and $|B|=\left\lceil\frac{|V(G)|}{2}\right\rceil$. (Later we show that most of our components have maximum or almost maximum allowable order.) Given that the components of $G-H$ are what is important, we can also prove the following lemma.

Lemma 6. Let $G$ be a graph with $|V(G)| \geqslant 3$, let $v$ be a vertex of $G$, and let $\mathcal{B}$ be the canonical line-bramble for $v$. If $H$ is a minimum hitting set for $\mathcal{B}$, then no edge of $H$ has both endpoints in the same component of $G-H$.

Proof. For the sake of a contradiction assume that both endpoints of an edge $e \in H$ are in the same component of $G-H$. Then consider the set $H-e$. By Lemma 5, $H-e$ is a hitting set of $\mathcal{B}$, since the vertex sets of the components of $G-H$ have not changed. But $H-e$ is smaller than the minimum hitting set $H$, a contradiction.

\section{PROOF OF THEOREM 1}

Let $G:=K_{n}$. When $n \leqslant 2$, Theorem 1 holds trivially, so assume $n \geqslant 3$. First, we determine a lower bound on the treewidth by considering a canonical line-bramble for $v$, denoted $\mathcal{B}$. Given that $K_{n}$ is regular, it suffices to choose a vertex $v$ of $K_{n}$ arbitrarily.

If $H$ is a minimum hitting set of a canonical line-bramble $\mathcal{B}$, label the components of $G-H$ as $Q_{1}, \ldots, Q_{p}$ such that $\left|V\left(Q_{1}\right)\right| \geqslant\left|V\left(Q_{2}\right)\right| \geqslant \ldots \geqslant\left|V\left(Q_{p}\right)\right|$. We refer to this as labeling the components descendingly.

Consider a pair of components $\left(Q_{i}, Q_{j}\right)$ where $i<j$ and the components are labeled descendingly. Call this a good pair if one of the following conditions hold:

1. $\left|V\left(Q_{i}\right)\right|<\frac{n}{2}-1$,

2. $n$ is even, $\left|V\left(Q_{i}\right)\right|=\frac{n}{2}-1, V\left(Q_{j}\right) \neq\{v\}$, and $v \notin V\left(Q_{i}\right)$.

Lemma 7. Let $G$ be a complete graph with $n \geqslant 3$ vertices, let $v$ be a vertex of $G$, let $\mathcal{B}$ be the canonical line-bramble for $v$, and let $H$ be a minimum hitting set of $\mathcal{B}$. If $Q_{1}, \ldots, Q_{p}$ are the components of $G-H$ labeled descendingly, then $Q_{1}, \ldots, Q_{p}$ does not contain a good pair.

Proof. Say $\left(Q_{i}, Q_{j}\right)$ is a good pair. Let $x$ be a vertex of $Q_{j}$, such that if $\left(Q_{i}, Q_{j}\right)$ is of the second type, then $x \neq v$. Let $H^{\prime}$ be the set of edges obtained from $H$ by removing the edges from $x$ to $Q_{i}$ and adding the edges from $x$ to $Q_{j}$. Then the components for $G-$ $H^{\prime}$ are $Q_{1}, \ldots, Q_{i-1}, Q_{i} \cup\{x\}, Q_{i+1}, \ldots, Q_{j-1}, Q_{j}-\{x\}, Q_{j+1}, \ldots Q_{p}$. By Lemma 5 , to ensure $H^{\prime}$ is a hitting set, it suffices to ensure that $V\left(Q_{i}\right) \cup\{x\}$ is sufficiently small, 
since all other components are the same as in $G-H$, or smaller. If $\left(Q_{i}, Q_{j}\right)$ is of the first type, then $\left|V\left(Q_{i}\right) \cup\{x\}\right|=\left|V\left(Q_{i}\right)\right|+1<\frac{n}{2}$. If $\left(Q_{i}, Q_{j}\right)$ is of the second type, then $\left|V\left(Q_{i}\right) \cup\{x\}\right|=\frac{n}{2}$, but it does not contain $v$. Thus, by Lemma $5, H^{\prime}$ is a hitting set. However, $\left|H^{\prime}\right|=|H|-\left|V\left(Q_{i}\right)\right|+\left|V\left(Q_{j}\right)\right|-1 \leqslant|H|-1$, which contradicts that $H$ is a minimum hitting set.

Lemma 8. Let $G, v, \mathcal{B}$ and $H$ be as in Lemma 7. Then $G-H$ has exactly three components.

Proof. Recall by Lemma 5, there is an upper bound on the order of the components of $G-H$. First, show that $G-H$ has at least three components. If $G-H$ has only one component, clearly this component is too large. If $G-H$ has two components and $n$ is odd, then one of the components must have more than $\frac{n}{2}$ vertices. If $G-H$ has two components and $n$ is even, it is possible that both components have exactly $\frac{n}{2}$ vertices; however, one of these components must contain $v$. Thus $G-H$ has at least three components. Now, assume $G-H$ has at least four components and label the components of $G-H$ descendingly. We show that these components contain a good pair, contradicting Lemma 7.

If $n$ is odd, there is a good pair of the first type when any two components have less than $\frac{n-1}{2}$ vertices. Thus, at least three components have order at least $\frac{n-1}{2}$. Then $|V(G)| \geqslant 3\left(\frac{n-1}{2}\right)+1>n$ when $n \geqslant 2$, which is a contradiction.

If $n$ is even, there is a good pair of the first type when any two components have less than $\frac{n}{2}-1$ vertices. Similarly to the previous case, $|V(G)| \geqslant 3\left(\frac{n}{2}-1\right)+1>n$, again a contradiction when $n>4$. If $n=4$ then each component is a single vertex. Take $Q_{i}, Q_{j}$ to be two of these components, neither of which contain the vertex $v$. Then $\left(Q_{i}, Q_{j}\right)$ is a good pair of the second type. Hence $G-H$ does not have more than three components, and as such it has exactly three components.

Lemma 9. Let $G, v, \mathcal{B}$ and $H$ be as in Lemma 7, and let the components of $G-H$ be labeled descendingly. If $n$ is odd then $\left|V\left(Q_{1}\right)\right|=\left|V\left(Q_{2}\right)\right|=\frac{n-1}{2}$ and $\left|V\left(Q_{3}\right)\right|=1$. If $n$ is even then $\left|V\left(Q_{1}\right)\right|=\frac{n}{2},\left|V\left(Q_{2}\right)\right|=\frac{n}{2}-1$ and $\left|V\left(Q_{3}\right)\right|=1$.

Proof. Lemma 8 shows that $G-H$ has exactly three components. By Lemma 7, $\left(Q_{2}, Q_{3}\right)$ is not a good pair. Hence $\left|V\left(Q_{1}\right)\right| \geqslant\left|V\left(Q_{2}\right)\right| \geqslant \frac{n-1}{2}$ when $n$ is odd, and $\left|V\left(Q_{1}\right)\right| \geqslant\left|V\left(Q_{2}\right)\right| \geqslant \frac{n}{2}-1$ when $n$ is even, or else there is a good pair of the first type. When $n$ is odd, it follows from Lemma 5 that $\left|V\left(Q_{1}\right)\right|=\left|V\left(Q_{2}\right)\right|=\frac{n-1}{2}$, and so $\left|V\left(Q_{3}\right)\right|=1$. When $n$ is even, however, $\frac{n}{2}-1 \leqslant\left|V\left(Q_{1}\right)\right|,\left|V\left(Q_{2}\right)\right| \leqslant \frac{n}{2}$. Since $Q_{3}$ is not empty, it follows that $\left|V\left(Q_{3}\right)\right|=1$ or 2 . If $\left|V\left(Q_{3}\right)\right|=1$, then $\left|V\left(Q_{1}\right)\right|=$ $\frac{n}{2},\left|V\left(Q_{2}\right)\right|=\frac{n}{2}-1$ and $\left|V\left(Q_{3}\right)\right|=1$, as required. Otherwise, $\left|V\left(Q_{1}\right)\right|,\left|V\left(Q_{2}\right)\right|=\frac{n}{2}-1$ and $\left|V\left(Q_{3}\right)\right|=2$. But then at least one of $Q_{1}, Q_{2}$ does not contain $v$, and $V\left(Q_{3}\right) \neq\{v\}$. Thus either $\left(Q_{1}, Q_{3}\right)$ or $\left(Q_{2}, Q_{3}\right)$ is a good pair of the second type, contradicting Lemma 7.

Lemma 10. Let $G, v, \mathcal{B}$ and $H$ be as in Lemma 7. Then $|H|=\left(\frac{n-1}{2}\right)\left(\frac{n-1}{2}\right)+(n-1)$ when $n$ is odd, and $|H|=\left(\frac{n-2}{2}\right)\left(\frac{n}{2}\right)+(n-1)$ when $n$ is even.

Proof. From Lemma 9 we know the order of the components of $G-H$. By Lemma 6 , $H$ is exactly the set of all edges between each pair of components, and since $G$ is complete there is an edge for each pair of vertices. From this it is easy to calculate $|H|$.

Lemma 10 and the Treewidth Duality Theorem imply: 
Corollary 11. Let $G$ be a complete graph with $n \geqslant 3$ vertices. Then

$$
\begin{aligned}
& \operatorname{pw}(L(G)) \geqslant \operatorname{tw}(L(G)) \\
& \quad=\operatorname{bn}(L(G))-1 \geqslant \begin{cases}\left(\frac{n-1}{2}\right)\left(\frac{n-1}{2}\right)+(n-2), & \text { if } n \text { is odd } \\
\left(\frac{n-2}{2}\right)\left(\frac{n}{2}\right)+(n-2), & \text { if } n \text { is even. }\end{cases}
\end{aligned}
$$

Now, to obtain an upper bound on $\operatorname{pw}(L(G))$, construct a path decomposition of $L(G)$. First, label the vertices of $G$ by $1, \ldots, n$. Let $T$ be an $n$-node path, also labeled by $1, \ldots, n$. The bag $A_{i}$, for the node labeled $i$, is defined such that $A_{i}=\{i j \in E(G)$ : $j \in V(G)\} \cup\{u w: u<i<w\}$. For a given $A_{i}$, call the edges of $\{i j \in E(G): j \in V(G)\}$ initial edges and call the edges of $\{u w: u<i<w\}$ crossover edges. (Note here these edges of $G$ are really acting as vertices of $L(G)$, but refer to them as edges for simplicity.)

Lemma 12. Let $G$ be a complete graph with $n \geqslant 3$ vertices. Then $\left(T,\left\{A_{1}, \ldots, A_{n}\right\}\right)$ is a path decomposition for $L(G)$ of width

$$
\begin{cases}\left(\frac{n-1}{2}\right)\left(\frac{n-1}{2}\right)+(n-2), & \text { if } n \text { is odd } \\ \left(\frac{n-2}{2}\right)\left(\frac{n}{2}\right)+(n-2), & \text { if } n \text { is even. }\end{cases}
$$

Proof. Each edge $u w$ of $G$ appears in $A_{u}$ and $A_{w}$ as an initial edge. Observe that $u w$ is in $A_{i}$ if and only if $u \leqslant i \leqslant w$, so the nodes indexing the bags containing $u w$ form a connected subtree of $T$. Finally, all of the edges incident at the vertex $u$ appear in $A_{u}$, and the same holds for $w$, so if two edges of $G$ are adjacent in $L(G)$, they share a bag.

Now determine the size of $A_{i}$. The bag $A_{i}$ contains $n-1$ initial edges and $(i-1)(n-i)$ crossover edges. So $\left|A_{i}\right|=(n-1)+(i-1)(n-i)$. This is maximized when $i=\frac{n+1}{2}$ if $n$ is odd, and when $i=\frac{n}{2}$ or $\frac{n+2}{2}$ if $n$ is even. From this it is possible to calculate the largest bag size, and hence the width of $T$.

Lemma 12 gives an upper bound on pw $\left(L\left(K_{n}\right)\right)$ and also on $\operatorname{tw}\left(L\left(K_{n}\right)\right)$. This, combined with the lower bound in Corollary 11, completes the proof of Theorem 1.

\section{REFERENCES}

[1] H. Bodlaender, A partial $k$-arboretum of graphs with bounded treewidth, Theor Comput Sci 209(1-2) (1998), 1-45.

[2] J. Díaz, M. Serna, and N. Wormald, Bounds on the bisection width for random $d$-regular graphs, Theor Comput Sci 382(2) (2007), 120-130.

[3] M. Grohe and D. Marx, On tree width, bramble size, and expansion, J Comb Theory Ser B 99(1) (2009), 218-228.

[4] D. Marx, Can you beat treewidth?, Theory Comput 6 (2010), 85-112. 
[5] B. Reed, Tree width and tangles: a new connectivity measure and some applications, Surveys in combinatorics, London Math. Soc. Lecture Note Ser., vol. 241, Cambridge Univ. Press, 1997, pp. 87-162.

[6] P. Seymour and R. Thomas, Graph searching and a min-max theorem for tree-width, J Comb Theory Ser B 58(1) (1993), 22-33. 